\title{
Neumonectomía en atelectasia masiva post infección por adenovirus
}

\author{
ANDRÉS KOPPMANN A.*, ANA MARÍA ESCOBAR C.* e ISABEL VALDÉS I.*
}

\section{Pneumonectomy in massive atelectasis post adenovirus infection}

We described the clinical and radiological evolution and pulmonary function tests in three patients aging 8 to 14 years old at present time. All of them suffered severe pneumonia caused by adenovirus infection during the infant period. During the acute viral disease, massive lung atelectasis refractory to treatment, determined the indication of pneumonectomy. All of the removed lungs presented bronchiectasis, atelectasis and fibrosis. In two children the medical condition improved after surgery, allowing to discontinue oxygen therapy. Wotwith standing, both of them had severe restrictive and obstructive limitation and signs of chronic damage at CTscan obstructive exacerbations decreased and the patients have been to carry out a normal life. The third patient has evolved with progressive clinical deterioration and pulmonary hypertension. The is under continuous oxygen therapy, because of extensive damage in the remaining lung. Being the pneumonectomy an exceptional procedure in children, in these patients the indication was due to medical treatment failure and progressive deterioration. Notwithstanding two children have shown, up to now, a satisfactory adaptation to this condition.

Key words: pneumonectomy, atelectasis, adenovirus.

\section{Resumen}

Se describen las características clínicas, la imagenología torácica y función pulmonar de tres pacientes (edades actuales: 8-14 años). Todos ellos cursaron con una neumonía grave por adenovirus en el período de lactante y cuya evolución se agravó por una atelectasia pulmonar masiva refractaria al tratamiento que determinó la indicación de neumonectomía. En los tres casos, el pulmón resecado presentaba atelectasia, bronquiectasias y fibrosis. Dos niños mejoraron su condición clínica posterior a la neumonectomía, logrando suspender el oxígeno adicional. A pesar de presentar una limitación ventilatoria restrictiva y obstructiva severa y una tomografía computarizada de pulmón con signos de daño crónico, han disminuido las exacerbaciones obstructivas y hacen vida normal. El tercer paciente ha evolucionado con deterioro clínico progresivo, requiere oxígeno adicional a permanencia y presenta hipertensión pulmonar, determinado por el extenso daño del pulmón remanente. Siendo la neumonectomía un procedimiento extremo en pediatría, a estos pacientes se les indicó por fracaso del tratamiento médico y empeoramiento del estado general. Dos niños han mostrado hasta ahora una adaptación satisfactoria a esta condición.

Palabras clave: Neumonectomía, atelectasia, infección por adenovirus.

* Unidad de Enfermedades Respiratorias, Hospital de Niños Roberto del Río.

Departamento de Pediatría Campus Norte, Facultad de Medicina, Universidad de Chile. 


\section{Introducción}

Las infecciones respiratorias agudas bajas por adenovirus constituyen una de las principales causas de enfermedad pulmonar crónica en pediatría, cuyo espectro abarca distintas entidades tales como hiperreactividad bronquial, bronquiectasias, atelectasias crónicas, bronquiolitis obliterante y pulmón hiperlúcido ${ }^{1-3}$. La neumonectomía es un procedimiento extremo en la práctica clínica y existen escasas publicaciones sobre el uso de este procedimiento en pacientes con daño crónico del pulmón remanente ${ }^{4}$. El objetivo de la presente comunicación es describir las características clínicas, la imagenología torácica y la función pulmonar de tres pacientes pediátricos que cursaron con una neumonía grave por adenovirus y cuya evolución se agravó por la presencia de una atelectasia pulmonar masiva refractaria al tratamiento que determinó la indicación de neumonectomía.

\section{Casos Clínicos}

\section{Paciente 1}

Escolar de 8 años, sexo femenino, con antecedentes de neumonía adquirida en la comunidad a los 2 meses de vida en la que se confirmó la presencia de adenovirus (ADV) por inmunofluorescencia. Evoluciona grave, requiriendo conexión a ventilación mecánica (VM) por 23 días. Permanece hospitalizada por 5 meses, siendo dada de alta a los 7 meses de vida con oxígenoterapia domiciliaria. Se realiza tomografía axial computarizada (TAC) de tórax que demuestra atelectasia del lóbulo superior derecho (LSD) y bronquiectasias cilíndricas en lóbulo inferior izquierdo. Entre los 10 meses y el año de vida se rehospitaliza en dos ocasiones por neumonía y síndrome bronquial obstructivo requiriendo ventilación mecánica en las dos hospitalizaciones. $\mathrm{Al}$ año y tres meses de vida durante una visita de rutina en policlínico de especialidad, se controla con una radiografía de tórax que evidencia una atelectasia masiva del pulmón izquierdo. Se realizan repetidas broncoaspiraciones mediante fibrobroncoscopía no lográndose la reexpansión pulmonar. El cintigrama ventilación-perfusión (Figura 1) muestra hipofunción pulmonar izquierda severa (ventilación pulmón derecho (PD): 83\%, pulmón izquierdo (PI): 17\%; perfusión PD: $86 \%$, PI: $14 \%$ ), debiendo ser neumonectomizada al año y siete meses de edad. El pulmón conservado presentaba una atelectasia residual del LSD. Evoluciona sin complicaciones post operatorias siendo dada de alta con oxígenoterapia nocturna. Dos meses más tarde se suspende el oxígeno adicional. Evoluciona bien, sin rehospitalización y sin hipertensión pulmonar. La TAC pulmonar 3 años post-neumonectomía evidencia dilatación compensatoria del pulmón remanente con atelectasia retráctil del LSD, engrosamiento intersticial peribronquial y áreas de mosaico leve en regiones basales (Figura 2). El estudio funcional realizado hace un año revela una limitación ventilatoria obstructiva y restrictiva severas con aumento significativo de la CVF y del $\mathrm{VEF}_{1}$ post-salbutamol. El estudio de volúmenes pulmonares mediante lavado de nitróge-

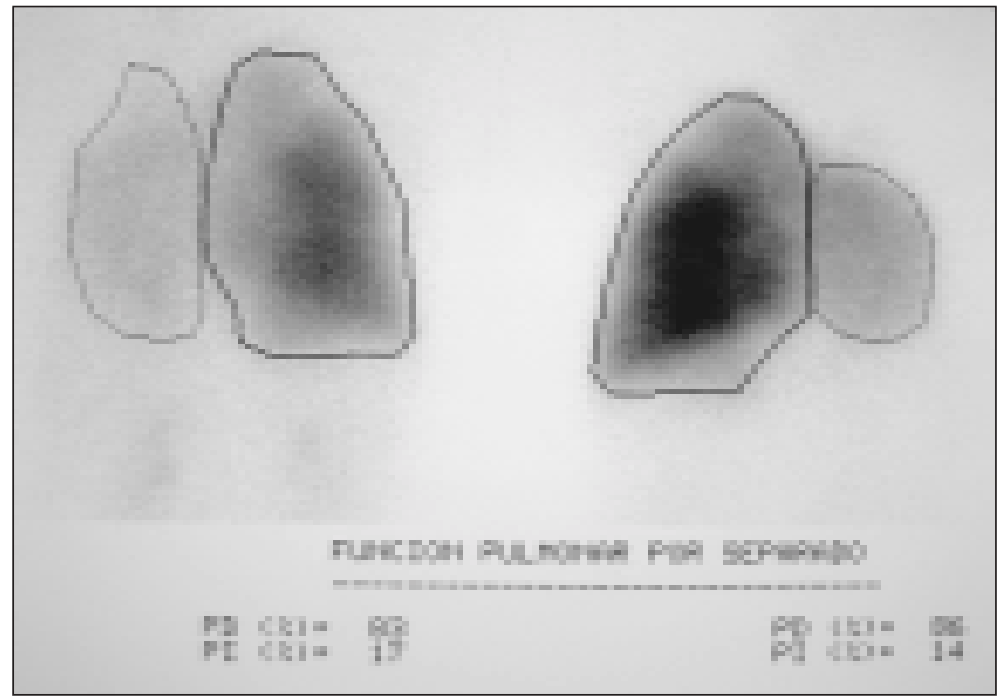

Figura 1. Cintigrama ventilaciónperfusión de la paciente 1 , muestra hipofunción pulmonar izquierda severa. A la izquierda, se indica la ventilación en $\%$ del pulmón derecho (PD) e izquierdo (PI) y a la derecha el de circulación. 


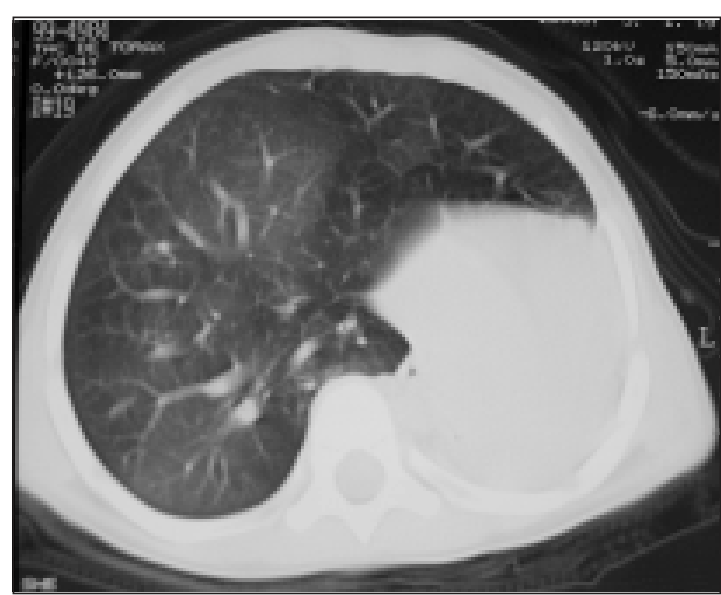

Figura 2. TAC pulmonar de la paciente 1, tres años postneumonectomía. Se observa importante dilatación compensatoria del pulmón derecho.

no muestra una capacidad pulmonar total (CPT) normal para el valor predictivo con un gran atrapamiento aéreo dado por un volumen residual (VR) aumentado. En la actualidad asiste al colegio y hace vida normal.

\section{Paciente 2}

Escolar de 8 años y un mes, sexo femenino, con antecedentes de haber sido recién nacido de pretérmino de 27 semanas, con peso de nacimiento de 980 gramos, que evoluciona con displasia broncopulmonar permaneciendo 98 días conectada a ventilación mecánica, siendo dada de alta a los 5 meses de edad cronológica con oxígenoterapia domicilaria. Se rehospitaliza an- tes del año de vida en tres oportunidades por cuadros bronquiales obstructivos sin requerir ventilación mecánica. Al año y un mes de vida presenta una bronconeumonía intrahospitalaria por ADV, que evoluciona en forma tórpida, con sobreinfección por virus sincicial respiratorio y bacteriana, requiriendo conexión a ventilación mecánica durante 17 días. En el curso de esta hospitalización presenta una atelectasia masiva del pulmón derecho, refractaria a broncoaspiración endoscópica. Se realiza TAC pulmonar que evidencia colapso pulmonar derecho total con bronquiectasias y pulmón izquierdo con compromiso intersticial segmentario en ambos lóbulos. La cintigrafía ventilación-perfusión (Figura 3) muestra exclusión funcional del pulmón derecho (ventilación PD: 6\%, PI: 94\%; perfusión PD: $2 \%$, PI: 98\%), debiendo realizarse neumonectomía al año y diez meses de vida. No presenta complicaciones post operatorias y es dada de alta con oxígenoterapia nocturna. Un año post-cirugía se suspende el oxígeno adicional. Evoluciona sin rehospitalizaciones y sin hipertensión pulmonar. La TAC pulmonar realizada a los 2 años post-neumonectomía, muestra gran expansión compensatoria del pulmón izquierdo, con áreas de mosaico leve y compromiso intersticial difuso. Se observa además crecimiento del pulmón remanente (Figura 4). Su espirometría actual revela una limitación ventilatoria obstructiva severa que mejora significativamente post salbutamol, combinada con una limitación restrictiva severa. No ha sido posible estudiar volúmenes pulmonares por falta de colaboración. En la actualidad asiste al colegio y hace vida normal.

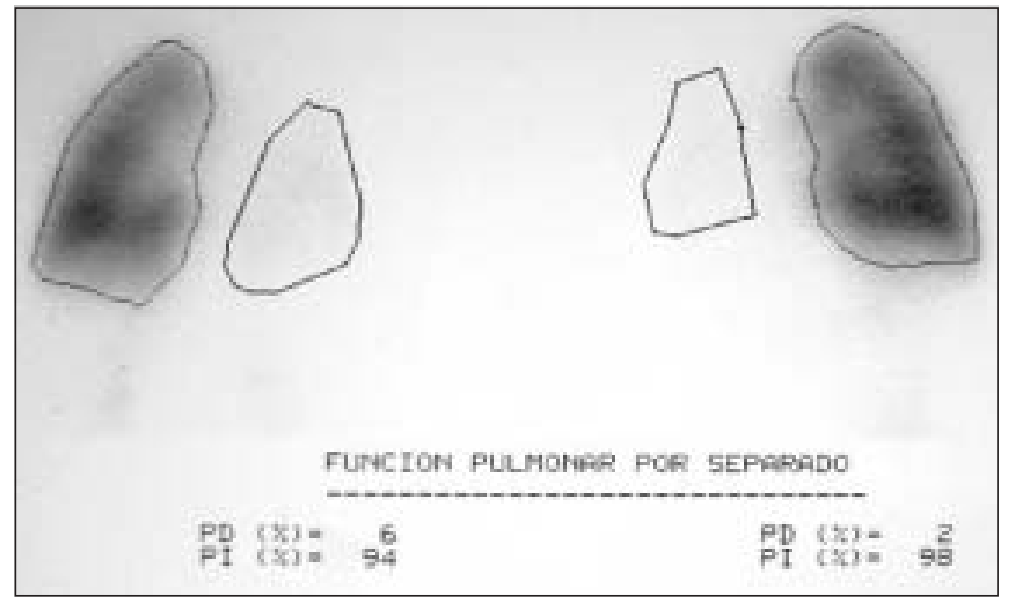

Figura 3. Cintigrama ventilación perfusión de la paciente 2, muestra exclusión funcional de pulmón derecho. PD y PI como en figura 1. 


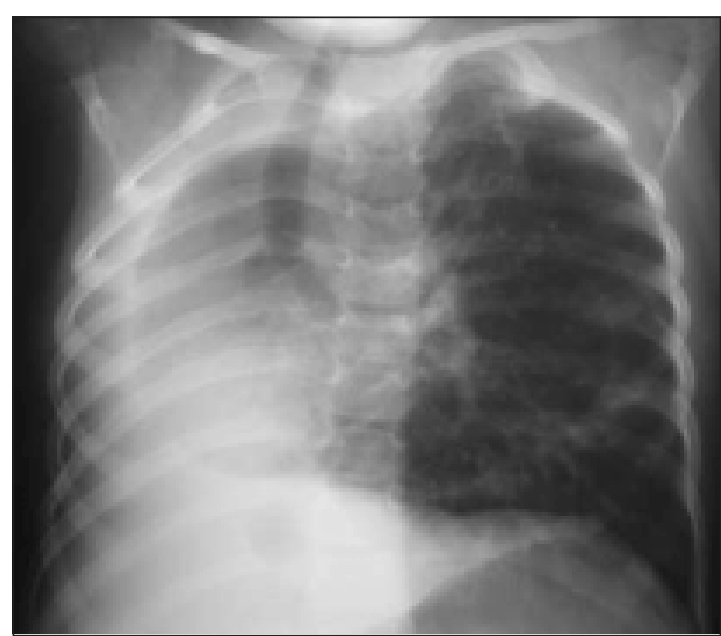

Figura 4. Radiografía de tórax de la paciente 2, cuatro años post neumonectomía. Se observa gran crecimiento compensatorio del pulmón izquierdo.

\section{Paciente 3}

Escolar de 14 años y 2 meses, sexo masculino, con historia de cuadros bronquiales obstructivos desde los 2 meses de vida. Al año y tres meses presentó una bronconeumonía intrahospitalaria por ADV serotipo $7 \mathrm{~h}$, requiriendo ventilación mecánica por 15 días; y permaneció hospitalizado durante 8 meses. Evolucionó con bronquiolitis obliterante y es dado de alta con oxigenoterapia domiciliaria. Reingresó a los 2 años y 5 meses de edad por atelectasia masiva del pulmón izquierdo, detectada en control rutinario que no logra ser reexpandida. Se efectúa TAC pulmonar que muestra colapso total de pulmón izquierdo y bronquiectasias bilaterales. La cintigrafía ventilación-perfusión demostró hipofunción pulmonar izquierda severa (ventilación PD: 89\%, PI: 11\%; perfusión PD: $83 \%$, PI: $17 \%$ ). Se realiza neumonectomía izquierda sin complicaciones post operatorias y es enviado a su casa con oxígenoterapia nocturna. En el curso de su evolución presenta rehospitalizaciones por neumonías recurrentes, constatándose además hipertensión pulmonar y escoliosis leve. La TAC pulmonar efectuada 8 años postneumonectomía, muestra dilatación compensatoria del pulmón derecho con herniación hacia el hemitórax izquierdo y bronquiectasias (Figura 5). En la actualidad requiere oxígenoterapia a permanencia y su espirometría muestra limitación ventilación obstructiva y restrictiva severas sin cambio significativo post-salbutamol.

El estudio histopatológico del pulmón reseca-

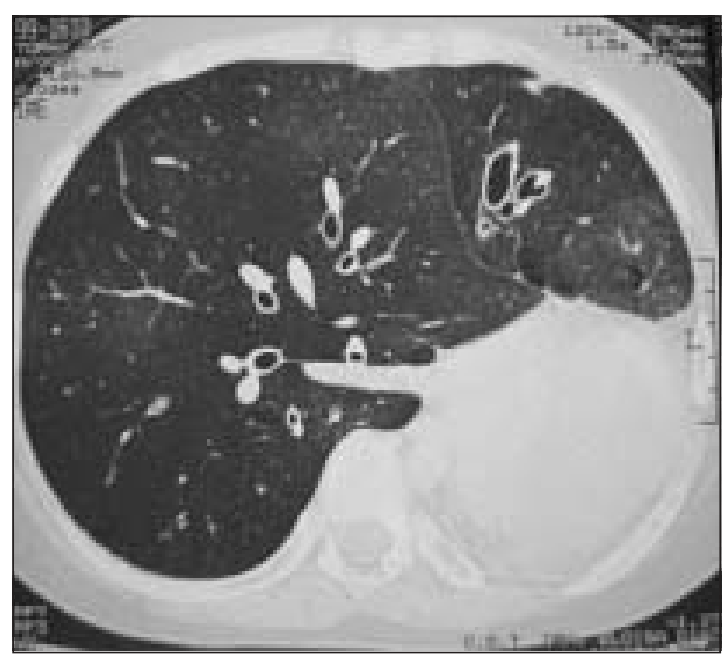

Figura 5. TAC pulmonar del paciente 3, ocho años post neumonectomía. Se aprecia dilatación de pulmón derecho con herniación hacia hemitórax izquierdo y bronquiectasias.

do en los tres casos descritos, demostró una severa alteración de la arquitectura normal, con extensas zonas de atelectasia, bronquiectasias saculares, hiperplasia de folículos linfoides y fibrosis intersticial y peribronquiolar.

En los tres pacientes se estudiaron otras causas de obstrucción bronquial y neumonías recurrentes. Estos exámenes incluyeron electrolitos en sudor, recuento de inmunoglobulinas, estudio de deglución y pH-metría esofágica que resultaron normales. En los tres casos además, tanto la serología para VIH como las baciloscopías fueron negativas. Se han realizado hasta la fecha evaluaciones cardiológicas periódicas descartando hipertensión arterial pulmonar en las dos niñas. En el tercer caso se ha documentado la existencia de hipertensión arterial pulmonar severa y un cor pulmonar a través de estudio ecocardiográfico y cateterismo cardíaco. Sólo en este niño además se ha detectado una escoliosis de carácter leve que se mantiene en control por el equipo de ortopedia. En la Tabla 1 se resumen las características clínicas de los tres niños y en la Tabla 2 se registran los valores espirométricos actuales.

\section{Discusión}

La neumonía grave por adenovirus se ha asociado a importantes secuelas pulmonares tales como hiperreactividad bronquial, bronquiectasias, atelectasias crónicas, bronquiolitis obliterante y 
Tabla 1. Características clínicas de los tres pacientes en estudio

\begin{tabular}{|c|c|c|c|}
\hline & $\begin{array}{c}\text { Paciente } 1 \\
8 \text { años }\end{array}$ & $\begin{array}{c}\text { Paciente } 2 \\
8 \text { años } 1 \text { mes }\end{array}$ & $\begin{array}{c}\text { Paciente } 3 \\
14 \text { años } 2 \text { meses }\end{array}$ \\
\hline Enfermedad previa & Sana & $\mathrm{DBP} *$ & $\mathrm{SBOR} * *$ \\
\hline Edad al momento de infección por ADV & 2 meses & 1 año 1 mes & 1 año 3 meses \\
\hline Lugar de adquisición de la infección & Comunitaria & Intrahospitalaria & Intrahospitalaria \\
\hline Edad al momento de la atelectasia & 1 año 2 meses & 1 año 3 meses & 2 años 5 meses \\
\hline Edad al momento de la neumonectomía & 1 año 7 meses & 1 año 10 meses & 2 años 7 meses \\
\hline Pulmón neumonectomizado & Izquierdo & Derecho & Izquierdo \\
\hline Estado del pulmón remanente & Atelectasia LSD & $\begin{array}{c}\text { Compromiso } \\
\text { intersticial }\end{array}$ & Bronquiectasias \\
\hline Complicaciones post operatorias inmediatas & No & No & No \\
\hline Complicaciones post operatorias tardías & No & No & Escoliosis \\
\hline Oxígenodependencia actual & No & No & $\mathrm{Si}$ \\
\hline Limitación ventilatoria actual & $\begin{array}{l}\text { Obstructiva y } \\
\text { restrictiva severa }\end{array}$ & $\begin{array}{l}\text { Obstructiva y } \\
\text { restrictiva severa }\end{array}$ & $\begin{array}{l}\text { Obstructiva y } \\
\text { restrictiva severa }\end{array}$ \\
\hline
\end{tabular}

Tabla 2. Valores espirométricos actuales de los tres pacientes en estudio

\begin{tabular}{|c|c|c|c|c|c|c|c|c|c|c|c|c|c|c|c|}
\hline & \multicolumn{5}{|c|}{$\begin{array}{l}\text { Paciente } 1 \\
8 \text { años }\end{array}$} & \multicolumn{5}{|c|}{$\begin{array}{c}\text { Paciente } 2 \\
8 \text { años } 1 \text { mes }\end{array}$} & \multicolumn{5}{|c|}{$\begin{array}{c}\text { Paciente } 3 \\
14 \text { años } 2 \text { meses }\end{array}$} \\
\hline & \multicolumn{2}{|c|}{ Basal } & \multicolumn{2}{|c|}{ Post BD ${ }^{* * *}$} & \multirow{2}{*}{ \% cambio } & \multicolumn{2}{|c|}{ Basal } & \multicolumn{2}{|c|}{ Post BD*** } & \multirow{2}{*}{ \% cambio } & \multicolumn{2}{|c|}{ Basal } & \multirow{2}{*}{\multicolumn{2}{|c|}{$\begin{array}{r}\text { Post BD** } \\
\text { \%pred* }\end{array}$}} & \multirow[t]{2}{*}{$\%$ cambio } \\
\hline & Actual & $\%$ pred $^{*}$ & Actual & $\%$ pred* & & Actual & $\%$ pred* & Actual & $\%$ pred* & & Actual & $\%$ pred* & & & \\
\hline CVF (L) & 0,39 & 42 & 0,55 & 59 & 41 & 0,37 & 42 & 0,44 & 49 & 19 & 1,23 & 43 & 1,48 & 51 & 20 \\
\hline $\mathrm{VEF}_{1}(\mathrm{~L})$ & 0,29 & 32 & 0,47 & 52 & 62 & 0,35 & 40 & 0,42 & 48 & 20 & 0,78 & 31 & 0,85 & 34 & 9 \\
\hline $\mathrm{VEF}_{1} / \mathrm{CVF}(\%)$ & 74 & & 86 & & 16 & 94 & & 96 & & 2 & 63 & & 58 & & -8 \\
\hline $\mathrm{FEF}_{25-75}(\mathrm{~L} / \mathrm{s})$ & 0,25 & 16 & 0,61 & 39 & 144 & 0,47 & 30 & 0,68 & 44 & 45 & 0,43 & 15 & 0,55 & 19 & 28 \\
\hline
\end{tabular}

* \% del predictivo (según Kundson et al)

** post broncodilatador

pulmón hiperlúcido ${ }^{1-3}$. Estudios de seguimiento de largo plazo han demostrado que alrededor del $20 \%$ de los niños que presentaron un daño pulmonar agudo por adenovirus, quedaron con algún tipo de secuela, siendo predominante en estos pacientes el antecedente de infección por serotipo $7 \mathrm{~h}^{1,5}$. La atelectasia masiva que presentaron nuestros pacientes durante los primeros meses posterior a la infección, demuestra la existencia de un gran daño anatómico. En el estudio histopatológico de los pulmones resecados se observa que este daño está dado fundamentalmente por la obliteración de los bronquíolos terminales por tejido fibroso concéntrico y por la intensa inflamación de las paredes bronquiales, dando origen a hipersecreción mucosa, tapones mucosos y bronquiectasias. Este tipo de lesiones explican en gran medida el porqué a pesar de broncoaspiraciones repetidas por vía endoscópica, no fue posible lograr la reexpansión de la atelectasia masiva en ninguno de los tres niños.

La neumonectomía es un procedimiento de excepción en pediatría y aunque se desconoce su prevalencia por la escasa información disponible en la literatura médica, ha ido disminuyendo 
fundamentalmente por el mejor control de la tuberculosis. En las series publicadas, las grandes indicaciones corresponden a las neumopatías necrotizantes, bronquiectasias, neumopatías supurativas y algunas malformaciones congéni$\operatorname{tas}^{6,7}$. La destrucción pulmonar condiciona un proceso inflamatorio crónico con reagudizaciones y reinfecciones frecuentes que alteran severamente la calidad de vida del paciente, su estado nutricional y favorecen complicaciones graves como hemoptisis masiva, infecciones fúngicas, etc. En tales casos la neumonectomía puede resolver esta situación. Sin embargo, su indicación debe ser siempre discutida en profundidad considerando las características clínicas y funcionales de cada paciente en particular. En niños neumonectomizados con pulmón remanente sano, la evolución y el pronóstico a largo plazo son buenos. Laros et $\mathrm{al}^{8}$, publicaron una serie de 130 pacientes neumonectomizados, la mayoría por tuberculosis, con un seguimiento promedio de 33 años. Noventa y ocho tenían $\mathrm{VEF}_{1}$ mayor o igual a $63 \%$ de la capacidad vital (CV) en su primera evaluación espirométrica y constituyeron el grupo a analizar. Cuarenta y tres pacientes fueron neumonectomizados antes de los 20 años de vida y dentro de este grupo la mejor adaptación se observó en los niños operados antes de los 5 años, en quienes los valores promedios de CPT y CV fueron $96,4 \%$ y $79,1 \%$ respectivamente del valor predictivo de los dos pulmones y el valor promedio de $\mathrm{VEF}_{1}, 72,3 \%$ de la CV. Ellos concluyen que en este grupo etario el mecanismo adaptativo fundamental sería el crecimiento compensatorio o hiperplasia del pulmón remanente y que a edades mayores la compensación estaría dada esencialmente por hipertrofia y dilatación ${ }^{8}$. En esta serie la mayoría de los pacientes estudiados durante el seguimiento hace vida normal en la actualidad.

La morbilidad y las complicaciones inmediatas post-neumonectomía pueden presentarse hasta en un $20 \%$ de los casos y éstas incluyen edema pulmonar en el pulmón remanente, neumonía, atelectasias, fistulas broncopleurales y herniación cardíaca. También se ha descrito el síndrome post-neumonectomía derecha que se presenta especialmente en lactantes y que consiste en la desviación del corazón y estructuras mediastínicas a la derecha, con rotación de estas últimas y fenómenos de compresión vascular del árbol bronquial izquierdo, llevando a broncomalacia, hiperinsuflación e infecciones recurrentes $^{9}$. En ninguno de los pacientes estudiados se observaron estas complicaciones. A largo plazo las complicaciones más temidas son las defor- midades esqueléticas, específicamente la escoliosis. Sin embargo, los estudios de seguimiento son muy escasos ${ }^{9}$. En una serie de 13 niños neumonectomizados seguidos por un promedio 5,2 años, Eren et $\mathrm{al}^{7}$ observaron que sólo seis pacientes desarrollaron escoliosis leve (ángulo de Coobs menor de $10^{\circ}$ ). Todos ellos habían sido intervenidos antes de los 7 años de vida. En nuestro grupo sólo un paciente ha desarrollado escoliosis, la cual es poco significativa, a pesar que los tres fueron neumonectomizados antes de los 3 años de edad. La recomendación no obstante, es un control ortopédico periódico y el uso de corset corrector según sea el caso.

Existen muy pocas publicaciones sobre neumonectomías en pacientes con daño crónico del pulmón conservado. Fregonese et $\mathrm{al}^{4}$, comunican el caso de un niño de 11 años portador del síndrome de Macleod/Swyer-James con bronquiectasias bilaterales, en el cual fue necesario realizar una neumonectomía del pulmón hiperlúcido debido a la broncorrea, las infecciones bacterianas recurrentes y el deterioro clínico progresivo que presentaba. En el seguimiento de 9 años, consignan una importante mejoría de la función pulmonar, así como también de su calidad de vida, haciéndose incluso independiente del uso de oxígeno. En nuestros pacientes se indicó neumonectomía no obstante la existencia de daño crónico en el pulmón contralateral, dado el riesgo vital inmediato que ellos presentaban. En dos casos fue posible suspender el oxígeno adicional poco tiempo después de la cirugía, como resultado de la reducción del shunt intrapulmonar condicionado por el pulmón fisiológicamente excluido. Durante el seguimiento realizado hasta ahora, hemos observado que a pesar de la limitación ventilatoria restrictiva y obstructiva severas que ellos presentan y los elementos propios de daño pulmonar crónico en las TAC de control (patrón en mosaico, bronquiectasias), las dos niñas no han requerido nuevas hospitalizaciones y sus exacerbaciones han disminuido. Se han mantenido sin necesidad de oxígeno adicional y asisten con normalidad al colegio. Ambas tienen una muy buena respuesta al broncodilatador, con mejoría significativa de su espirometría lo que hace suponer una hiperreactividad bronquial asociada.

El caso del $3^{\text {er }}$ paciente es diferente. El daño extenso del pulmón remanente ha ocasionado un deterioro progresivo de su condición clínica, con requerimiento de oxigenoterapia permanente y desarrollo de hipertensión arterial pulmonar, lo que hace que su pronóstico a corto plazo sea sombrío. 
En suma, podemos decir que la neumonectomía representa un procedimiento extremo en medicina y su indicación debe ser valorada en forma individual considerando las características del paciente y la situación clínica y funcional en la que éste se encuentre. En nuestros pacientes fue necesario realizarla a pesar del daño en el pulmón contralateral, dado el riesgo vital que ellos presentaban. En el transcurso de su evolución hemos observado una adaptación satisfactoria en dos de ellos, no obstante el significativo deterioro en su función pulmonar. Es importante destacar también que en dos de nuestros casos la adquisición del adenovirus fue nosocomial, lo que hace necesario tomar medidas a fin de mejorar las técnicas de aislamiento en enfermos respiratorios.

\section{Bibliografías}

1.- TORRES G, LARRAÑAGA C, LOZANO J, UBILLA C, KAJON A, AVENDAÑO L F. Evolución clínica a largo plazo de la infección respiratoria aguda baja por adenovirus. Acta Microbiológica 1996; 7 supl 1: 14-16.

2.- ZHANG L, IRION K, KOZAKEWICH H, REID L, CAMARGO J J, DA SILVA N, et al. Clinical course of postinfectious bronchiolitis obliterans. Pediatr Pulmonol 2000; 29: 341-50.

3.- CHANG A B, MASEL J P, MASTERS B. Post infectious bronchiolitis obliterans: clinical, radiological and pulmonary function sequelae. Pediatr Radiol 1998; 28: 23-9.

4.- FREGONESE L, GIROSI D, BATTISTINI E, FREGONESE B, RISSO F M, BAVA G L, et al. Clinical, physiologic and roentgenographic changes after pneumonectomy in a boy with Macleod/Swyer-James syndrome and bronchiectasis. Pediatr Pulmonol 2002; 34: 412-6.

5.- PALOMINO M A, LARRAÑAGA C, AVENDAÑO L F. Hospital acquired adenovirus $7 \mathrm{~h}$ infantile respiratory infection in Chile. Pediatr Infect Dis J 2000; 19: 52731.

6.- BLYTH D F, BUCKELS N J, SEWSUNKER R, SONI M A. Pneumonectomy in children. Eur J Cardiothorac Surg 2002; 22: 587-94.

7.- EREN S, EREN M N, BALCI A E. Pneumonectomy in children for destroyed lung and the long-term consequences. J Thorac Cardiovasc Surg 2003; 126: 574-81.

8.- LAROS C D, WESTERMANN C J. Dilatation, compensatory growth, or both after pneumonectomy during childhood and adolescence. J Thorac Cardiovasc Surg. 1987; 93: 570-6.

9.- LEZAMA-DEL VALLE P, BLAKELY M, LOBE T E. Long-term consequences of pneumonectomy done in children. In: Deslauriers J, Faber LP, editors. Chest Surgery Clinics of North America, Philadelphia, PA: Saunders, 1999. pp 485-95.

Correspondencia a:

Dr. Andrés Koppmann A.

Profesor Zañartu 1085, Independencia

Teléfonos: 4791150 - 5446375 (fonofax)

9-2340289

E-mail: andreskoppmann@yahoo.com 